\title{
Vorberich t.
}

Der dritte Jahresbericht über die Fortschritte der Physik in Jahre 1847 erscheint etwas verspätet, wofür wir als Entschuldigung die politischen Ereignifse anfülıren mülsen, indem einige Mitarbeiter am Jahresberichte ihrer gesellschaftlichen Stellung nach keine Mufse für wissenschaftliche Arbeiten finden konnten. Wir hoffen den nächsten Jahrgang desto schneller folgen lassen zu können, um so mehr als die physikalischen Untersuchungen des Jahres 1848 der Zahl nach weit geringer sind als die der vorhergehenden drei Jahre.

Leider ist es uns nur zum Theil gelungen, die im zweiten Jahrgange bemerkbaren Licken auszufüllen. Der praktische Theil einiger Capitel, die Hydraulik und Aëromechanik bleibl unbearbeitet, und wir werden uns auch fernerhin darauf beschränken bei diesen Abschnitten nur die in den physikalischen Schriften enthaltene Literatur aufzuführen. Der Anhang zur Elektricitätslehre, welcher die elektrische Telegraphie behandelt, mufste fortbleiben, da der Berichterstatter Herr Lieutenant W. Stemans keine Zeit gewinnen konnte; im nächsten Jahrgange soll der Bericht für 1847 und 1848 erfolgen, diesmal geben wir nur die Literatur. Uebrigens werden auch für diesen $\mathbf{A b s c h n t t}$ so wie für die Galvanoplastik die rein technischen Aufsätze künftig nur in der Literatur aufgeführt werden. Für die physikalische 
Geographie konnte noch kein Mitarbeiter gewonnen werden. Dagegen dürfen wir uns um so mehr freuen, durch die Güte des Hrn. Buys-Ballot in Utrecht in diesem Jahrgange den meteorologischen Bericht von 1845 bis 1847 haben geben zu können, als der ursprüngliche Berichterstatter für Meteorologie, Hr. Dr. Mahlmann der Gesellschaft plötzlich durch den Tod entrissen wurde. Hrn. Lıмолт in München endlich verdanken wir in einem Refèrate über Erdmagnetismus eine wichtige Bereicherung des vorliegenden Jahrganges.

Lücken im Einzelnen sind ohne Zweifel noch manche vorhanden, was seinen hauptsächlichen Grund darin hat, dafs wir nur von sehr wenigen Monographien haben Kenntnifs erhalten können. Unsre, im zweiten Jahresberichte ausgesprochene Bitte hat geringen Erfolg gehabt, indem bis jetzt nur sehr wenige gelehrte Gesellschaften und Herausgeber von periodischen Zeitschriften einen Tausch der Schriften mit unsrem Jahresberichte angenommen haben, noch weniger uns Monographien zugesendet wurden.

Indem wir unsern Dank für die uns gemachten Mittheilungen aussprechen, wiederholen wir hiermit unsre frühere Aufforderung im Interesse der Wissenschaft, und in eignen Interesse der Schriftsteller, deren Arbeiten durch den Jahresbericht gewifs eine allgemeinere Verbreitung erhalten. Es muls noch bemerkt werden dafs wir alle physikalischen Arbeiten auch nachträglich berücksichtigen die in den Bereich der Jahresberichte fallen, also die seit 1845 erschienen sind.

Von nächsten Jahrgange an wird im Vorberichte ein Verzeichnifs über die versendeten und empfangenen Schriften geführt werden.

Nachrichten über die physikalische Gesellschaft.

Im Laufe des Jahres 1847 sind aus der Zahl der Mitglieder aus der physikalischen Gesellschaft geschieden:

Die Herren: Becker, Böнm, v. Pochнammer. 
Aufgenommen wurden:

Die Herren: v. Bruchhausen, Heidel, Jacobi, Kuhn, LAmont, Liebe, Ludivig, Mensing, Soltmann II., Vögeli.

Mitglieder der Gesellschaft waren am Ende des Jahres 1847 :

Hr. Dr. D'Arrest in Leipzig. Hr. Conservator Dr. Lamont

- Dr. W. BeEtz.

- Mechan. Bötricher.

- Dr. E. du Bors - REYMond.

- Brauns.

- Prof. Dr. E. Brücke in Wien.

- Dr. Brünnow in Bilk.

- Lieuten. Dr. v. Bruchinusen.

- Dr. C. Brunner (Sohn) in Bern.

- Mechan. Duve.

- Dr. Eisenstein.

- Dr. T. Ewald.

- Prof. Dr. v. Feilitzsch in Greifswalde.

- Mechan. HaLsKe.

- Heider.

- Dr. W. Heintz.

- Dr. Helmholtz.

- Dr. D'Heureuse.

- Hptm. JАсов.

- Jungr.

- Prof. Dr. G. Karsten in Kiel.

- v. Kıréewsky in Petersburg.

- Prof. Dr. H. Knoblauch in Marburg.

- Dr. A. KRöNig.

- Prof. Dr. KuHN in München. in München.

- Prof. Dr. Langberg in Christiania.

- Mechan. Leonhardt.

- Lieuten. Liebe.

- Prof. Dr. Ludwrg in Marburg.

- Dr. Mahlahann.

- Mechan. Martins.

- Lieuten. Mensing.

- Lieuten. v. Morozowicz.

- Mechan. Pistor.

- H. Poselger.

- Direkt. Quetelet in Brüssel.

- Medic. R. Dr. Quincke.

- Prof. Dr. Radicke in Bonn.

- Rohrbeck.

- Lieuten. Siemens.

- Dr. Soltmann I.

- Soltmann II.

- Dr. G. SPörer in Prentzlau.

- Dr. Traube I.

- Traube II.

- Dr. Vögeli.

- Dr. Wüchter.

- Dr. Wiedemans.

- Dr. Wilhęluy. 
Im dritten Jahre des Bestehens der physikalischen Gesellschaft wurden folgende Originaluntersuchungen der Mitglieder in den Sitzungen der Gesellschaft vorgetragen:

1847.

8. Januar. H. Kмовца бсн. Neues Verfahren die Mannigfaltigkeit der Wärmestrahlen zu ermitteln.

22. Jan. G. Kansten. Ueber das Leitungsvermögen der Schwefelmetalle. Pogc. Ann. LXXI. 239.

- Ueber die Verdunstungsgränze des Quecksilbers. eb. 245.

5. Febr. E. Bnückx. Ueber die Bestimmung des specifischen Gewichtes der Milch durch das Aräometer und das Tausendgranfläschchen. Mǘc. Arch. f. Anat. u. Phys. 1847. 409.

19. Febr. E. Bnücse. Ueber das Leuchten der Menschenaugen. Ebendas. 225. 479 .

5. März. v. EnцA cr. Ueber das Verhalten der organischen Elementartheile im polarisirten Lichte. Ebendas. 313.

E. Внüскв. Ueber die Faserung der Krystallinse des Auges. Der menschliche Augapfel. Berlin bei Reimer, 1847. $4^{\circ}$ p. 27.

19. März. E. DU Bors-Rermond. Ueber das Gesetz des Muskel - und Nervenstrounes. Untersuchungen über thierische Elektricität. Bd. I. Bd. II. Abth. I.

H. Knosicuch. Ueber den Durchgang der strahlenden Wärme durch Krystalle in verschiedenen Richtungen. Pose. Annalen. LXX. 1.

16. April. G. Kansten. Pyroelektricität des derben Boracits. Poge. Ann. LXXI. 243.

- Ueber elektrische Staubfiguren. ib. 244.

- Ueber galvanoplastisches irisirendes Kupfer. ib. 246.

Lodwis. Ueber den Druck der unter verschiedenen Umständen auf die Arterienwände ausgeübt wird. MüLL. Arch. 1847. 242.

30. April. A. Kä̈nis. Theorie des Anorthoskopes von Plateau. Ungedruckt.

H. Kмовцатст. Ueber die Methoden zum Nachweise der Beugung der Wärmestrahlen. Berl. Ber. 1846. S. 311.

W. Bzetz. Darstellung Norru'scher Farbenringe und einfarbiger Platten auf galvanischem Wege. 
14. Mai. Е. Виüскк. Ueber einen eigenthümlichen Ring an der Krystallinse der Vïgel. MǘL. Arch. 1847. 477.

E. Du Boxs-Reymond. Ueber den Cylinder als den Rotationskürper rom geringsten Widerstande gegen die Elektricität bei gleicliem Stoffe gleicher Länge u. Grundfläche. Unters. II. Bd. I. Abth. 79.

Jorer. Ueber die Formation des am 25sten Mai gefallenen Hagels. Ungedruckt.

9. Juli. H. Knomlauch. Ueber die Polarisation der Wärme durch Nrcoz'sche Prismen, Glasspiegel und (1lassätze. Pogo. Ann. LXXIV. 161.

E. Baücex. Mittheilung des Hrn. Ragona-Scra über physiologische Ergäazıngsfarben. Dies. Ber. p. 189.

23. Juli. H. Kжosnauch. Untersuchungen über die Doppelbrechung der Wärme. Pogg. Ann. LXXIV. 1.

Hzқmozz. Ueber das Princip der Erhaltung der Kraft. Berlin bei Reimer. 1847. $8^{\circ}$.

G. Spörer. Ueber die Unhaltbarkeit der MüpízR'schen Anuahme einer Centralsonne. Ungedruckt.

6. August. E. do Bors-Rermond. Starker elektrischer Stroun in den Darmmuskeln des Schlei's. Unters. Bd. II. Abthl. I. S. 200 .

- Ueber den Strom im contraktilen Gaumorgane der Cyprinoiden. Ebendas. S. 198.

20. Aug. H. Kмовцauch. Ueber die auf Doppelbrechung und Polarisation der Wärmestrahlen berulıenden Erscheinungen. Poge. Ann. LXXIV. 177.

v. Bruchinusen. Die Gleiclıungen der Flugbahn rotirender Geschosse. Berlin, Posen und Bromberg bei Mittler. 1847. $8^{\circ}$.

15. Oct. E. Bnücxz. Ueber ein Mittel die verschiedenen Wege anschaulich zu machen, welche eine schwingende Saite beschreibt je nachdem sie angestrichen worden ist. Ungedruckt.

29. Oct. Н. Kмовцатсн. Gesetze der Beugung der Wärinestrahlen. Poge. Ann. LXXIV. 9.

12. Nov. Wücнтеn. Ueber gemeinschaftlich mit Hrn. OscнАтz angestellte Untersuchungen die Porzellan-Bildung betreffend.

Hвьмноцтz. Untersuchungen über die Wärmeentwicklong bei Zusamunenziehung ausgeschnittener Muskeln kaltbliitiger Thiere. Mürtxn's Archiv 1848. 144.

26. Nov. E. Do Bors-Rermond. Ueber einen Multiplikator zur Un- 
tersuchung des Nervenstromes. Unters. Bd. II. Abthl. 1. 477 .

G. Kıяснноғт. Ueluer das elektrodynamische Gleichgewicht in einem Körper. Poge. Ann. LXXII. 497.

W. Siemens. Ueber den Widerstand der Erde gegen elektrische Ströme.

10. Dec. - Ueber das Verhältnifs des Widerstandes der Elektromagnete zun Widerstand der iibrigen Kette zur Erzielung des Maximuıs des Magnetismus.

Kiel den 10ten Mai 1849.

Prof. Dr. G. Karsten. 